\title{
Compuestos orgánicos persistentes en Colombia: cuantificación y diagnóstico para pesticidas organoclorados
}

\section{Persistentorganic pollutants in Colombia: quantification and diag- nosis for organochlorine pesticides}

César Augusto García Ubaque

Ingeniero civil, magíster en Ingeniería Ambiental, Ph.D. en Ingeniería, profesor asociado de la Facultad Tecnológica de la Universidad Distrital Francisco José de Caldas, Bogotá D. C., Colombia. Contacto: cagarciau@udistrital.edu.co

\section{Juan Carlos García Ubaque}

Médico, magíster en Salud Pública, Ph.D. en Salud Pública, profesor titular de la Facultad de Medicina de la Universidad Nacional de Colombia, Bogotá D. C., Colombia. Contacto: jcgarciau@unal.edu.co

\section{Martha Lucía Vaca Bohórquez}

Psicóloga, especialista en Salud Ocupacional, especialista en Derecho Ambiental, magíster en Business Administration, consultora independiente, Bogotá D.C., Colombia. Contacto: ml.vaca@uniandes.edu.co

Fecha de recepción: 5 de febrero del 2014

Fecha de aceptación: 15 de agosto del 2014
Clasificación del artículo: estudio de caso Financiamiento: Programa de las Naciones Unidas para el Desarrollo (PNUD)

DOI: http://dx.doi.org/10.14483/udistrital.jour.tecnura.2015.1.a011

Palabras clave: compuestos orgánicos persistentes, plaguicidas, ecotoxicidad, inventarios.

Keywords: pesticides, persistent organic pollutants, ecotoxicity, inventories.

\section{RESUMEN}

Como parte del cumplimiento de los compromisos de Colombia con el Convenio de Estocolmo sobre Contaminantes Orgánicos Persistentes (COP), el país llevó a cabo la identificación y cuantificación de existencias de plaguicidas organoclorados, con el fin de actualizar y consolidar la información sobre sitios de almacenamiento y suelos contaminados. Se utilizó la metodolo- gía propuesta por el Programa de las Naciones Unidas para el Desarrollo (PNUD), que cubrió las siguientes etapas: a) consulta de información secundaria disponible en inventarios anteriores; b) revisión de las actividades relacionadas con las etapas del ciclo de vida de estos productos; c) ubicación de bodegas y sitios de destrucción o enterramiento; y d) visita a una muestra de los lugares identificados. Se encontró que el país tiene $159812 \mathrm{~kg}$ de existencias de DDT almacenado y 


\section{estudio de caso}

se estimó que existen $88629 \mathrm{~m}^{3}$ de suelo contaminado por plaguicidas COP. Se pudo identificar un amplio subregistro de las cantidades de pesticidas utilizadas y de sitios contaminados, no solo en el país, sino también en inventarios de otros países de la región, lo que refleja que los resultados reportados son parciales y pueden presentarse focos de contaminación no identificados. Es importante que ante esta situación se intensifiquen actividades de investigación e innovación para el tratamiento de estos residuos y tierras contaminadas, pero también en las áreas de producción agrícola y protección de cultivos.

\section{ABSTRACT}

To meet Colombia commitments with Stockholm Convention on persistent organic pollutants (POPs), the country carried out identification and quantification of organochlorine pesticide stocks,

\section{INTRODUCCIÓN}

La problemática relacionada con el uso de pesticidas tiene una doble cara. Por un lado, su uso permite mayores rendimientos en el cultivo de alimentos $\mathrm{y}$, consecuentemente, mejora las condiciones de abastecimiento tanto en el ámbito local como en el regional. Según Falconer y Hodge (2000), la producción mundial de alimentos disminuiría aproximadamente un $30 \%$ sin el uso de estas sustancias. Sin embargo, es innegable que su sobreutilización ha ocasionado graves daños al ambiente y a la salud de las personas. Es importante plantear un balance entre la protección del medio ambiente y la reducción del uso de pesticidas con la necesidad de optimizar la producción agrícola.

Es importante tener en cuenta que en la región andina Colombia es uno de los países con mayor consumo de pesticidas (García-Santos, Scheibeny in order to update and consolidate information on storage sites and contaminated soils. Methodology proposed by the United Nations program for development (UNDP) was used and covered the following stages: (a) consultation of secondary information available in previous inventories, (b) review activities related to these products life cycle, (c) location of warehouses and sites of destruction or burial and (d) visit a sample of identified sites. Colombia has $159812 \mathrm{~kg}$ of DDT in stock and it was estimated $88629 \mathrm{~m}^{3}$ of contaminated soil by POPs pesticides. It were unable to identify a vast underreporting quantities of pesticides used and contaminated sites, not only in the country, but in inventories in other countries of the region; reflecting that the reported results are partial and contamination from unidentified sources may occur. It is important to intensify activities of research and innovation not only for wastes and contaminated soils treatment, but for agricultural production and crop protection.

Binder, 2011), principalmente en cultivos de algodón (45\%), maíz, arroz y papa (estos tres con el 45\% de participación) (Machado, 1998). Como respuesta al incremento de la demanda, en la década de los años setenta, el país comenzó a producir toxafeno, aldrin y DDT. Hacia la década de los noventa, esta actividad fue totalmente suspendida, para cumplir con normas de la legislación nacional e internacional.

Como parte de los compromisos adquiridos por el país con la firma del Convenio de Estocolmo sobre Contaminantes Orgánicos Persistentes (COP), se llevó a cabo la identificación y cuantificación de existencias de plaguicidas organoclorados. El alcance de este inventario fue actualizar y consolidar la información relacionada con las existencias en el país de nueve plaguicidas COP: aldrin, dieldrin, endrin, clordano, heptacloro, hexaclorobenceno, mirex, toxafeno y DDT (Ministerio 


\section{estudio de caso}

de Ambiente, Vivienda y Desarrollo Territorial, 2007). Los objetivos específicos fueron: a) consolidar el inventario nacional de plaguicidas COP, mediante la revisión, la evaluación y la complementación de información secundaria disponible; b) recopilar y evaluar datos de producción, uso, importación y exportación de plaguicidas COP; y c) consolidar la información nacional sobre existencias, enterramientos y posibles sitios contaminados con estas sustancias.

\section{METODOLOGÍA}

La metodología utilizada para este inventario siguió los lineamientos planteados por el PNUD para la aplicación el Convenio de Estocolmo (PNUD, 2005). Una primera etapa consistió en consultar inventarios previos de plaguicidas obsoletos. Luego se recopiló y analizó la información secundaria relacionada con importaciones de plaguicidas COP y las actividades relacionadas con las diferentes etapas del ciclo de vida de estos productos. Además, se ubicaron bodegas y sitios de destrucción de plaguicidas en el país que generaron material de empaque contaminado o productos obsoletos por devoluciones; asimismo, se ubicaron instalaciones asociadas con el uso de plaguicidas COP como: pistas de fumigación, almacenes agrícolas y granjas de experimentación. Finalmente, se realizaron visitas a una muestra de estos sitios, correspondientes a los siguientes grupos: a) empresas estatales, b) agremiaciones y asociaciones y c) pistas de fumigación y empresas de agroquímicos.

\section{RESULTADOS}

Las tablas 1 a 5 muestran los resultados obtenidos en el inventario.

En la tabla 4 se presenta el total de existencias de plaguicidas COP almacenados y de suelo contaminado en el país.

Tabla 1. Material de residuos contaminados

\begin{tabular}{|l|c|l|}
\hline \multicolumn{1}{|c|}{ Material } & Cantidad & \multicolumn{1}{c|}{ Observaciones } \\
\hline Canecas plásticas de tapa ancha de 200 litros & 8 & $\begin{array}{l}\text { Canecas sobre estibas de madera, en regular estado, } \\
\text { mal tapadas, algunas con aros reventados. Contamina- } \\
\text { das con residuos de methilparathion y toxafeno, mez- } \\
\text { cladas con agua lluvia. }\end{array}$ \\
\hline Canecas metálicas de 200 litros & 66 & $\begin{array}{l}\text { Oxidadas y con la cubierta superior corroída y rota, } \\
\text { ingreso de agua lluvia. }\end{array}$ \\
\hline Cartón & $1 \mathrm{~m}^{2}$ & Apilamiento de cartón contaminado a la intemperie. \\
\hline $\begin{array}{l}\text { Contenedores tipo big-bag de polipropileno. } \\
\text { Capacidad: } 1 \mathrm{~m}^{3}\end{array}$ & 60 & $\begin{array}{l}\text { Contienen bolsas plásticas y de papel, madera y retal } \\
\text { de tambores metálicos. Todos los materiales contami- } \\
\text { nados y a medio quemar. Están a la intemperie. }\end{array}$ \\
\hline $\begin{array}{l}\text { Contenedores tipo big-bag de polipropileno. } \\
\text { Capacidad: } 1 \mathrm{~m}^{3}\end{array}$ & ND & $\begin{array}{l}\text { Bolsas rotas que contienen bolsas plásticas y de } \\
\text { papel, madera y retal de tambores metálicos. Todos } \\
\text { contaminados y a medio quemar, a la intemperie, inva- } \\
\text { didas por maleza. }\end{array}$ \\
\hline $\begin{array}{l}\text { Materiales varios como: guantes, manguera, } \\
\text { cascos }\end{array}$ & ND & $\begin{array}{l}\text { Guantes quemados, cascos, pedazos de bolsas y } \\
\text { canecas plásticas negras semidestruidas. }\end{array}$ \\
\hline $\begin{array}{l}\text { Bodegas y construcciones con pisos en ce- } \\
\text { mento y colectores perimetrales de agua lluvia, } \\
\text { con agua }\end{array}$ & ND & $\begin{array}{l}\text { Construcciones semidestruidas, sin techo o con te- } \\
\text { chos destruidos, con paredes y pisos contaminados. } \\
\text { Se observan grietas en algunos sectores y partes ex- } \\
\text { ternas con hundimientos. }\end{array}$ \\
\hline
\end{tabular}

Fuente: Ministerio de Ambiente, Vivienda y Desarrollo Territorial (2007). 


\section{estudio de caso}

Tabla 2. Existencias almacenadas de plaguicidas

\begin{tabular}{|l|l|l|l|}
\hline \multicolumn{1}{|c|}{ Producto } & \multicolumn{1}{c|}{ Cantidad } & \multicolumn{1}{c|}{ Unidades } & \multicolumn{1}{c|}{ Ubicación } \\
\hline DDT & 21920 & $\mathrm{~kg}$ & Bogotá, Cundinamarca \\
\hline DDT & 1890 & $\mathrm{~kg}$ & Cartagena, Bolívar \\
\hline DDT & 135402 & $\mathrm{~kg}$ & Mariquita, Tolima \\
\hline DDT & 600 & $\mathrm{~kg}$ & Puerto Inírida, Guainía \\
\hline Total & 159812 & $\mathrm{~kg}$ & \\
\hline
\end{tabular}

Fuente: Ministerio de Ambiente, Vivienda y Desarrollo Territorial (2007).

Tabla 3. Estimativo de suelo contaminado con plaguicidas COP

\begin{tabular}{|l|l|l|l|l|}
\hline \multicolumn{1}{|c|}{ Elemento } & \multicolumn{1}{|c|}{$\begin{array}{c}\text { Tipo de } \\
\text { producto }\end{array}$} & Cantidad & $\begin{array}{c}\text { Cantidad } \\
\text { estimada } \\
\text { identificados } \\
\text { de suelo } \\
\text { contaminado } \\
\left(\mathbf{m}^{3} \mathbf{)}\right.\end{array}$ \\
\hline $\begin{array}{l}\text { Plaguicidas COP } \\
\text { almacenados }\end{array}$ & DDT & $159812 \mathrm{~kg}$ & 4 & 5 \\
\hline $\begin{array}{l}\text { Plaguicidas COP } \\
\text { enterrados }\end{array}$ & Varios & Desconocida & 7 & 88160 \\
\hline $\begin{array}{l}\text { Suelo contaminado } \\
\text { por plaguicidas COP }\end{array}$ & Varios & Desconocida & 12 & 464 \\
\hline $\begin{array}{l}\text { Plaguicidas obsoletos } \\
\text { almacenados }\end{array}$ & Varios & $40440 \mathrm{~kg} \cdot \mathrm{L}$ & 29 & $\mathrm{ND}$ \\
\hline $\begin{array}{l}\text { Plaguicidas obsoletos } \\
\text { enterrados }\end{array}$ & Varios & Desconocida & 5 & ND \\
\hline Total & & & & 88629 \\
\hline
\end{tabular}

Fuente: Ministerio de Ambiente, Vivienda y Desarrollo Territorial (2007).

Tabla 4. Total de plaguicidas COP almacenados y suelo contaminado

\begin{tabular}{|l|l|}
\hline Producto: DDT & $159812 \mathrm{~kg}$ \\
\hline Estimación de suelo contaminado & $88629 \mathrm{~m}^{3}$ \\
\hline
\end{tabular}

Fuente: Ministerio de Ambiente, Vivienda y Desarrollo Territorial (2007).

No se pudieron identificar existencias significativas de otros plaguicidas diferentes a DDT, por lo cual solo se presentan los registros y las evidencias de esta sustancia.

Con el fin de comparar estos resultados con los de otros países de la región, se recopiló la infor- mación sobre existencias de DDT reportada por inventarios realizados por otros países como Chile (Conama, 2005), Ecuador (Ministerio del Ambiente, 2004), Perú (Conam, 2006) y Venezuela (Ministerio del Ambiente, 2006). En la figura 1 se puede apreciar la comparación de estos resultados con los datos reportados por Colombia. 


\section{| estudio de caso}

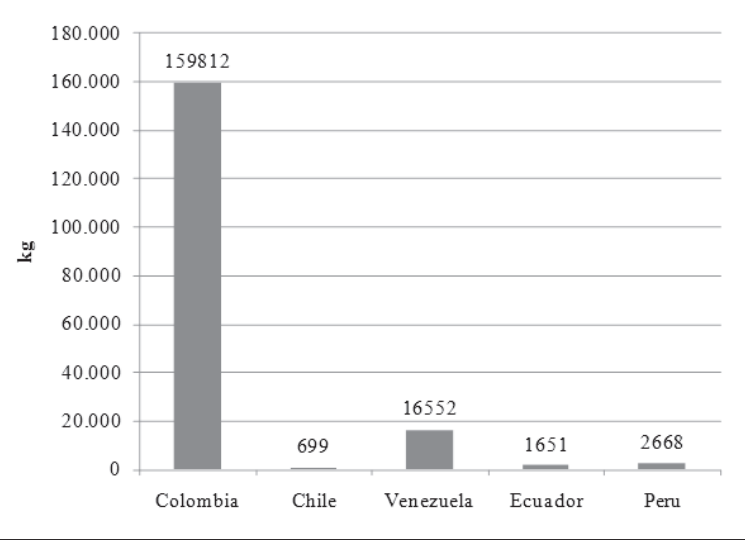

Figura 1. Existencias de DDT reportadas por países de la región

Fuente: elaboración propia.

\section{DISCUSIÓN}

De acuerdo con los resultados obtenidos en el inventario y su comparación con los registros presentados por los países vecinos, Colombia es de lejos el país que reporta mayores existencias de DDT en la región, lo cual es coherente con lo planteado por García-Santos, Scheiben y Binder (2011) sobre el mayor consumo de plaguicidas de nuestro país con respecto a los consumos en Suramérica. Sin embargo, estas diferencias también pueden deberse a subregistros en estos países y a que aun cuando existe una metodología propuesta por el PNUD, la presentación de los resultados de cada país difiere sustancialmente de acuerdo con la información disponible y la interpretación del equipo que realizó el trabajo.

Con respecto a los resultados encontrados específicamente en Colombia, durante el proceso de recolección de información se encontró que en algunas de las fuentes consultadas, tanto del sector privado como del público, existe desconocimiento sobre el tema y sobre los compromisos de Colombia con relación a los COP y al Convenio de Estocolmo, situación que plantea importantes re- tos sobre la manera en que el país puede mejorar sus estrategias para la erradicación de existencias de pesticidas COP y sitios contaminados; ya que existe un importante campo de acción para realizar acciones de mejora en términos de educación $\mathrm{y}$ entrenamiento.

Como lo plantean Falconer y Hodge (2000), más que instrumentos de mercado o de comando y control, se requiere fomentar la investigación y la innovación en el tema de protección de cultivos que generen soluciones económicamente rentables y que permitan un cambio de actitud tanto en los productores agrícolas como en consultores y tomadores de decisiones en esta área. Asimismo, es fundamental analizar las conductas a pequeña escala o a nivel micro (granjas/cultivos) para evaluar patrones de producción, requerimientos, intensidad, etc., con el fin de proponer políticas agrícolas pertinentes que realmente provean soluciones efectivas a la producción de alimentos de una manera sostenible y priorizar estrategias de educación y entrenamiento. Aunque se puede transferir tecnologías agrícolas exitosas en otras regiones, se deben ajustar a las condiciones locales, lo cual plantea retos de trabajo comunitario interdisciplinario más que de aplicación de recomendaciones de expertos.

Teniendo en cuenta las características de persistencia, poca movilidad y ecotoxicidad de los plaguicidas COP, que además fueron usados de manera intensiva y sobredosificada y eliminados mediante prácticas no adecuadas, el principal problema del país relacionado con estas sustancias es la existencia de suelos contaminados. Colombia debe desarrollar capacidades para remediación por este tipo de sustancias, ya que su mayor experiencia ha sido el manejo de la contaminación por derrame de hidrocarburos (Ministerio de Ambiente, Vivienda y Desarrollo Territorial, 2008). Algunas alternativas que se han venido estudiando es el uso de microorganismos para reducir las cantidades de pesticidas y rehabilitar los servicios ambientales 


\section{estudio de caso}

de estas tierras (Betancur, 2013; Mosquera y Peñuela, 2009), con índices de eficiencia entre 75y $95 \%$, pero dada la complejidad química de estas sustancias, es necesario intensificar la investigación para hallar soluciones más eficientes (Arbeli, 2009).

Para generar estrategias integrales a esta problemática se requiere la sensibilización y la generación de ambientes de confianza entre todas las partes involucradas tanto del sector público como del privado, mediante mesas de trabajo que incluyan: empresas, institutos de investigación, entidades del gobierno y otros, para desarrollar acciones conjuntas y coordinadas que permitan la eliminación de existencias y sitios contaminados con pesticidas COP y el desarrollo de nuevas tecnologías de protección de cultivos ambientalmente sostenibles.

\section{REFERENCIAS}

Arbeli, Z. (2009). Biodegradación de compuestos orgánicos persistentes (COP): el caso de los bifenilos policlorados (PCB). Acta Biológica Colombiana, 14 (1), 57-88.

Betancur, B. (2013). Biorremediación de suelo contaminado con el pesticida 1,1,1-Tricloro-2,2'BIS (p-clorofenil) etano (DDT) mediante protocolos de bioestimulación (tesis de grado de Maestría en Biotecnología). Universidad Nacional, Sede Medellín.

Consejo Nacional del Ambiente (Conam) (2006). Inventario nacional de plaguicidas COP. Lima: CONAM.

Comisión Nacional del Medio Ambiente (Conama) (2005). Plan Nacional de Implementación para la Gestión de los COP's. Santiago de Chile: Conama.

Falconer, K. y Hodge, I. (2000). Using Economic Incentives for Pesticide Usage Reductions: Responsiveness to Input Taxation and Agricultural Systems. Agricultural Systems, 63 (3), 175-194.

García-Santos, G., Scheiben, D. y Binder, C. (2011). The Weight Method: A New Screening Method for Estimating Pesticide. Chemosphere, 82 (11), 1571-1577.
Machado, A. (1998). La industria de agroquímicos y la agricultura colombiana. Bogotá: Asociación Nacional de Industriales (Andi).

Ministerio de Ambiente, Vivienda y Desarrollo Territorial (2007). Consolidación del inventario de plaguicidas COP. Bogotá: Ministerio de Ambiente, Vivienda y Desarrollo Territorial.

Ministerio de Ambiente, Vivienda y Desarrollo Territorial (2008). Plan Nacional de Aplicación para Plaguicidas en el Marco del Convenio de Estocolmo. Recuperado el 22 de noviembre de 2013, de http://www. minambiente.gov.co/documentos/3027 plan_accion_plaguicidas_consulta.pdf

Ministerio del Ambiente (2004). Inventario de Plaguicidas COPs en el Ecuador. Quito: Ministerio del Ambiente.

Ministerio del Ambiente (2006). Informe preliminar de inventarios de COPs en la República Bolivariana de Venezuela. Caracas: Ministerio del Ambiente.

Mosquera B., R. y Peñuela M., G. (2009). Biodegradación del malatión utilizando microorganismos nativos de suelos agrícolas. $R e$ - 


\section{estudio de caso}

vista Colombiana de Ciencias Pecuarias, 22 (2), 189-198.

Programa de las Naciones Unidas para el Desarrollo (2005). Guía para el desarrollo de un plan nacional de aplicación del Convenio de Estocolmo, Anexo 2. Ginebra: PNUD. 